\title{
Study of 3D Footprint Analysis Testing System
}

\author{
Peng Jin ${ }^{1, a}$, Wu Xing ${ }^{2, b}$, Pan Nan ${ }^{3, c^{*}}$ \\ ${ }^{1,2,3}$ Faculty of Mechanical \& Electrical Engineering, \\ Kunming University of Science \& Technology, \\ Kunming, China \\ alucas_faith@foxmail.com, ${ }^{b}$ xingwu@aliyun.com, ${ }^{c *} 15808867407 @ 163 . c o m$
}

Keywords: LabVIEW; Image mosaic; Laser ranging; 3D footprint analysis

Abstract. In order to narrow the range of suspects and clear direction of investigation quickly, combined laser ranging sensor, computer control and image technology, a 3D footprint analysis testing system was designed and development based on LabVIEW. The system design concept and embryonic form were all introduced in detail. Actual test experiments show the effectiveness of the system.

\section{Introduction}

The 3D footprint is the object of the interaction between the foot and the ground, and the object of the foot is in the shape of the object. The three-dimensional footprint is complete and fully reflects the full contact part of the appearance of the structure, but also intuitive and comprehensive reflection of the people's movement habits [1]. It can assist public security investigators to narrow the scope of the investigation, and also can be a strong evidence in the case proceedings [2].

Feature extraction of the 3D footprint normally by using the computer 3D surface modeling at home and abroad, there is a lack of unified inspection standard or excessive dependence on professional equipment and professional experience [3][4].

In view of the above problems, based on LabVIEW platform we design and develop a 3D footprint analysis testing system with laser ranging sensor, high-definition camera and an electric control slide hardware module.

\section{System Design}

\section{Overall System Block Diagram}

According to the demand analysis, the system can be divided into four modules: initialization and calibration, image acquisition and processing, feature extraction, analysis and database. System level block diagram is shown in Figure 1

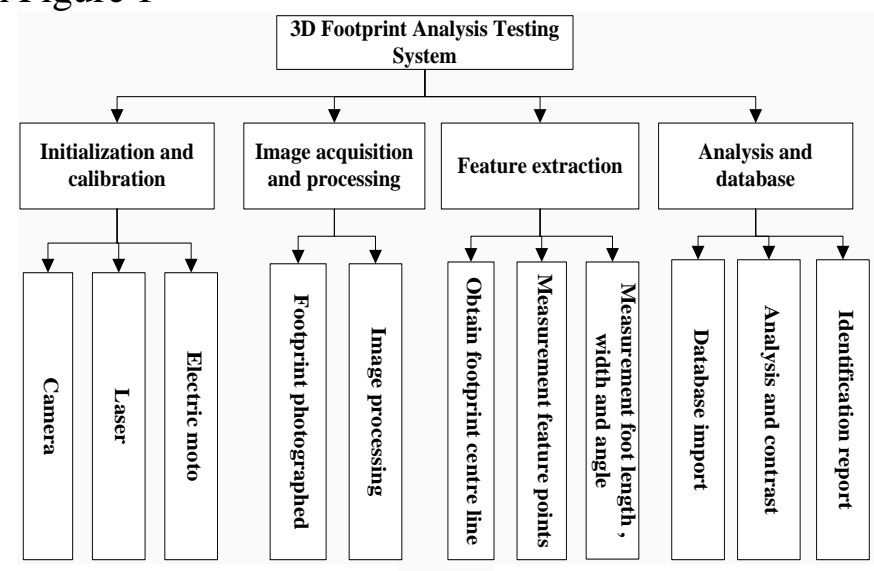

Fig. 1

\section{System Flow and Sub Modules}

1) After the calibration, collect the image, then the image is cut out and the effective feature region is extracted. 
2) After the image processing, firstly obtaining the footprint center line, set up a coordinate system. The laser sensor will automatically measure the range of the footprint's feature points. Then calculates the footprint's seven characteristic index (steep, semi steep, hallux steep, up angle, down angle, foot length and foot width).

3) The information will be saved to the corresponding database, analysis and comparison of footprint feature parameters according to the algorithm, thereby reducing or locked the suspect range, and finally gives the corresponding footprint inspection appraisal report. The whole process of the system is shown in figure 2.

4) Put the footprint model into the $3 \mathrm{D}$ footprint box, then running the system, judging left/right foot, and then configure the camera, laser and the motor.

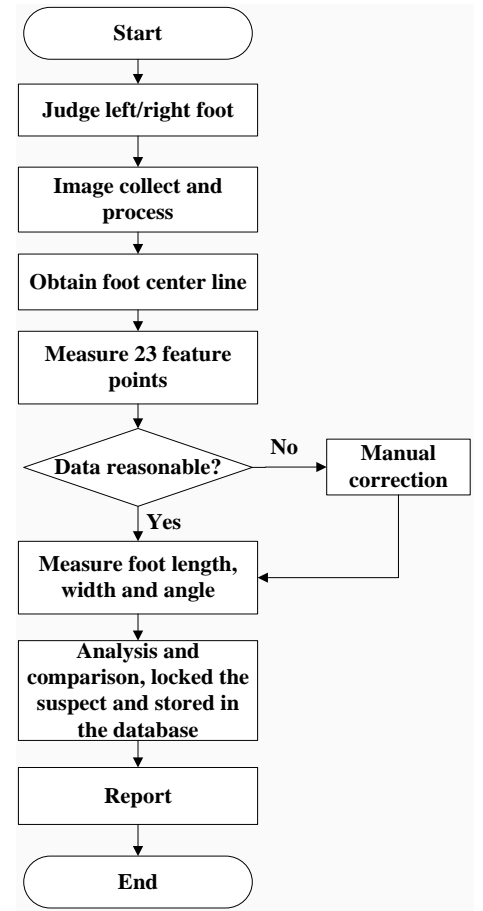

Fig. 2

\section{Software Implementations}

The system software is based on LabVIEW platform, with the DSC and Vision toolkit for development, the main interface of the system is shown in Figure 3

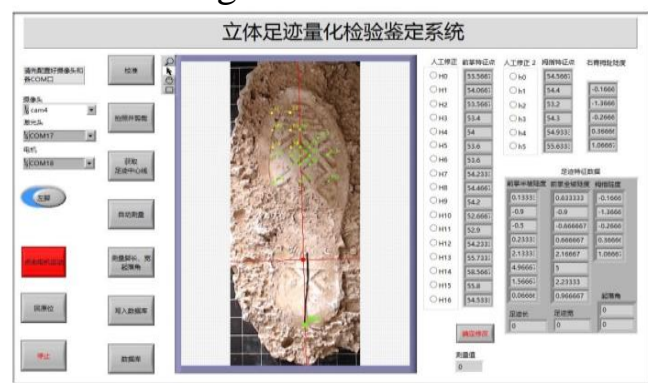

Fig. 3

\section{Initialization and Calibration}

Initialization and calibration module is an automatic control process of the stepping motor, camera and laser ranging sensor. Hardware including two-phase 42 series stepper motor, STC89C52 enhanced 51 SCM, Aluminum rails, Wenglor CP24MHT80 laser sensor and Logitech camera [5]. The software uses the NI-IMAQdx module under the Vision toolkit to control the camera, and the use of simple and low cost serial communication control progress of the motor and laser sensor [6][7]. The initialization and calibration procedures are shown in Figure 4. 


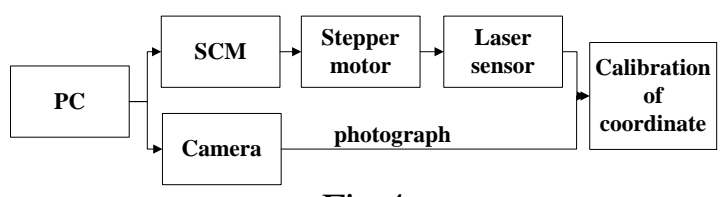

Fig.4

\section{Image Acquisition and Processing}

The image acquisition and processing module mainly uses the NI-IMAQdx control camera under the Vision toolkit, collects the photos of the stereo footprint model, and uses the Extract IMAQ function to achieve the initial rotation and cutting of the image, highlighting the special region of footprint, which lays the foundation for the subsequent feature extraction .

The concrete interface is shown in Figure 5.

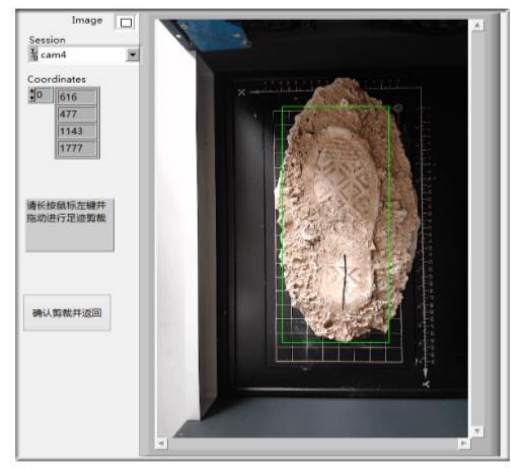

Fig.5

\section{Feature Extraction}

The feature extraction of the footprint is especially important in the process of the whole solid footprint analysis, which directly determines the final test results.

The 3D footprint analysis testing system present in this paper, which is a combination of computer automatic control and image technology, greatly reduces the difficulty and workload of the investigators.

Measurement of characteristic data:

Operating system, click automatic measurement button, the motor will drive the laser head automatic measuring above 23 points coordinates in the $\mathrm{Z}$ axis distance data, and display the whole slope steepness, semi slope steepness and hallux steepness on the front panel. If the individual point data is abnormal, carried out the artificial intervention and the individual outliers can be corrected. Then measure the up angle, down angle, foot length and foot width. Coordinate system as shown in figure 6

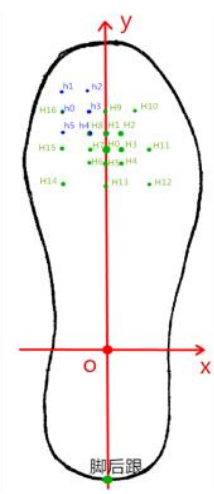

Fig.6

\section{Analysis and Database}

Through the collection and measurement, we can get the characteristic parameters of the crime person and suspects. Make the following definitions:

$$
\begin{aligned}
& A_{1}^{2}=\sum_{j=1}^{8}\left(g_{o j}-g_{i j}\right)^{2} \\
& A_{2}^{2}=\sum_{j=1}^{8}\left(g_{o j}^{\prime}-g_{i j}^{\prime}\right)^{2} \\
& A_{3}^{2}=\sum_{j=1}^{5}\left(d_{o j}-d_{i j}\right)^{2} \\
& A_{4}=\left|\alpha_{0}-\alpha_{i}\right|
\end{aligned}
$$




$$
\begin{aligned}
& A_{5}=\left|\beta_{0}-\beta_{\mathrm{i}}\right| \\
& \mathrm{A}_{6}=\left|\mathrm{I}_{0}-\mathrm{I}_{\mathrm{i}}\right| \\
& \mathrm{A}_{7}=\left|\mathrm{K}_{0}-\mathrm{K}_{\mathrm{i}}\right|
\end{aligned}
$$

After a lot of experiments, the 7 indicators of the characteristics of the 3D footprint are subject to normal distribution, and the threshold value of each index can be determined by using the mathematical statistics and the experimental data. By comparing the measured data and the theoretical threshold, we can determine the attribution of the trail, so as to achieve the purpose of determining or excluding the suspect. Seven indicators reference threshold as shown in Table 1.

Table 1

\begin{tabular}{|c|c|c|c|c|c|c|c|}
\hline Indicators & $\mathrm{A}_{1}^{2}$ & $\mathrm{~A}_{2}^{2}$ & $\mathrm{~A}_{3}^{2}$ & $\mathrm{~A}_{4}$ & $\mathrm{~A}_{5}$ & $\mathrm{~A}_{6}$ & $\mathrm{~A}_{7}$ \\
\hline Threshold & 45 & 25 & 22 & 8 & 8 & 11 & 54 \\
\hline Reliable probabilty & \multicolumn{6}{|c|}{ Greater than or equal to $99 \%$} \\
\hline
\end{tabular}

The measurement and calculation of the data will be in the form of a table in the database, the database can be added, delete, query and other operations. Finally output the "footprint test report".

\section{Experiments}

In order to verify the accuracy and practicality of the system, simulate the crime scene, and actual collect the 3D footprint plaster model, put it into the footprint box and running the system for test.

The footprint characteristic parameters of this system are shown in Table 2.

Table 2

\begin{tabular}{|c|c|c|c|c|c|c|c|}
\hline Indicators & Steep & Semi steep & Hallux steep & Down angle & Up angle & Foot length & Foot width \\
\hline Figures & 7.82 & 25.0 & 20.5 & 7.47 & 6.53 & 5.1 & 1.1 \\
\hline
\end{tabular}

\section{Summary}

This paper designs and develops a 3D footprint analysis and testing system with the accurate ranging of laser sensor and computer image and data analysis technology based on LabVIEW platform. Experimental results verify the practicality and accuracy of the system, which shows that the system can assist investigators to conduct a quantitative analysis of the three-dimensional footprint characteristics, but there are also a number of issues need to be improved:

1) The extraction and calculation accuracy of the footprint characteristic parameters have a higher requirement for the reduction of the previous three dimensional footprint models.

2) Continue adding measurement and calculation parameters of foot back palm area's feature points.

3) Also need to set up a perfect three-dimensional footprint sample network database and a unified inspection standard.

\section{References}

[1] Junliang Han. Trace inspection. China democracy and Law Press, 2007.6.

[2] Lei Wang. Role of footprints in Case Investigation. Legal and Social, 2008,(8), pp.165-166.

[3] Yinghui Yang. Advances in three-dimensional footprint Extraction Method. Yunnan Police Officer Academy, 2004,(1), pp.103-105.

[4] S. Mikkonen, V. Suominen, P. Heinonen. Use of footwear impressions in crime scene investigations assisted by computerized footwear collection system. Forensic Science International, 1996, (82), pp.67-79.

[5] Nan Pan, Yi Liu. A fast automatic acquisition instrument for 3D footprint. China: CN204465744U,2015-07-08.

[6] Jiangfang Xia, Shifu Zhao. Virtual instrument and the realization of serial communication between single chip microcomputer and single chip computer. Mechanical engineering and automation, 2006,(2), pp.136-137.

[7] Mang Guo, Lianqing Zhu, Xiaoquan Han. Design of CMOS camera image acquisition system based on LabVIEW. Automation application, 2014,(3), pp.15-16. 